\title{
THE NUMERICAL - EXPERIMENTAL ANALYSIS OF ABLATIVE LASER CLEANING NICKEL-BASED SUPERALLOY
}

\author{
Krzysztof Kosiuczenko \\ Military University of Technology, Faculty of Mechanical Engineering \\ Kaliskiego Street 2, 00-908 Warsaw, Poland \\ tel.: +48 22 683-72-01, fax: +48 22 683-93-55 \\ e-mail:kkosiuczenko@wat.edu.pl \\ Wojciech Napadtek \\ Military University of Technology, Faculty of Mechanical Engineering \\ Kaliskiego Street 2, 00-908 Warsaw, Poland \\ tel.: +4822683-73-48 \\ e-mail:wnapadlek@wat.edu.pl
}

\begin{abstract}
The results of simulation the laser ablation cleaning for the surface of samples made for nickel-based superalloy ŻS6K-WI. this material is use to produce the first-degree blades of aircraft engine turbines. The laboratory investigations were used pulse neodymium laser Nd: YAG and laser ytterbium Nd: Y3Al5O12. In parallel with the experiment was performed numerical simulation of ablation process. The simulation results were used for selection of appropriate laser parameters (duration, pulse form and the course of pulse in time, power density). the short time of the phenomenon of ablation process ( $10 \mathrm{~ns})$, the authors have chosen the finite element method (FEM) contained in the LS-Dyna. Since it is known that, the rate of thermal deformation is very large, for modelling the material properties of the material model was used Thermal-Elastic-Plastic. So chosen material model allows the dependence of material parameters ( $E, \alpha, v, R e, E T, C p, k)$ on temperature.

Scheme of laser ablation, diagram of laser power density, physical model, identification depth of the ablation, area where the temperature exceeded the melting temperature, in successive moments of loading are presented in the paper.
\end{abstract}

Keywords: superalloy ŻS6K-WI, laser ablation cleaning, numerical simulation MES

\section{Numerical simulation of ablation}

Due to the complexity of the microscopic phenomena, which occur during thermal ablation was the problem to make suitable selection of calculation and software method that will enable simulate all or the majority of the phenomena (such as molecular motion and phase transitions) and to analyze the material at the macroscopic level (for example, determine the state of stresses and strains in the sample). Given the short time of occurrence of phenomena ( $10 \mathrm{~ns})$, the authors of the article chose the finite element method (FEM) contained in the LS-Dyna [1].

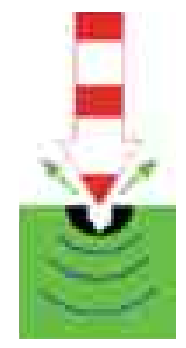

Fig. 1. Scheme of laser ablation 


\section{Modelling of laser interactions with materials}

The most important and the most difficult subject in the development of a numerical model was simulation the load - measures laser impulse (Fig. 2). The energy contained in the laser impulse depends on the laser power I ( $t$ ) and duration time $t$ Most of this energy is absorbed by the sample material.

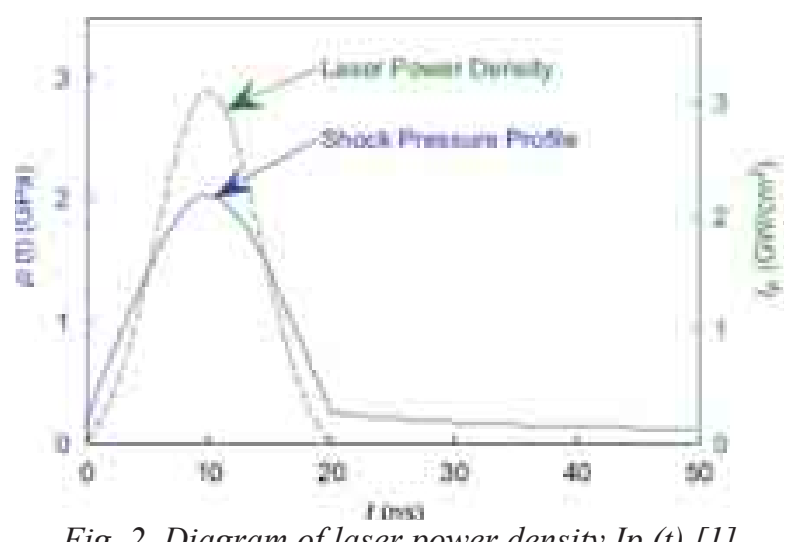

Fig. 2. Diagram of laser power density Ip (t) [1]

The absorption properties of $\mathrm{Ap}(\mathrm{t})$ also depend on time. For the purpose of numerical analysis, the authors assumed that the absorption coefficient has a constant value equal to 0.9.

$$
I_{p}(t)=A_{p}(t) I(t) \text {, }
$$

where:

I ( t) - the nominal laser power density, $\mathrm{W} / \mathrm{m}^{2}$,

Ip (t) - absorbed laser power density, $\mathrm{W} / \mathrm{m}^{2}$,

Ap (t) - absorption coefficient, which was adopted in accordance with [1].

Laser pulse parameters were calculated from the formulas and the work [2]. The depth of absorption can be estimated using the formula 2 [2]:

$$
l s=A p \lambda / 4 \pi=0.14 \mu m .
$$

The computed depth was taken into account in the construction of mesh model, FEA was used to estimate the thickness of the surface layer elements. Due to the complexity of physical phenomena, the numerical model considered the most important thermodynamic factors (Fig. 3):

- heating surface heat flow $\Phi(\mathrm{t})=1012 \mathrm{~W} / \mathrm{m}^{2}$,

- sample initial temperature $\mathrm{T}_{\mathrm{p}}=20^{\circ} \mathrm{C}$,

- constant temperature of the sample support $\mathrm{T}(\mathrm{t})=20^{\circ} \mathrm{C}$,

- cooling of the upper surface of the sample by forced convection for $\mathrm{T} \infty=20^{\circ} \mathrm{C}$,

- lack of heat in other areas.

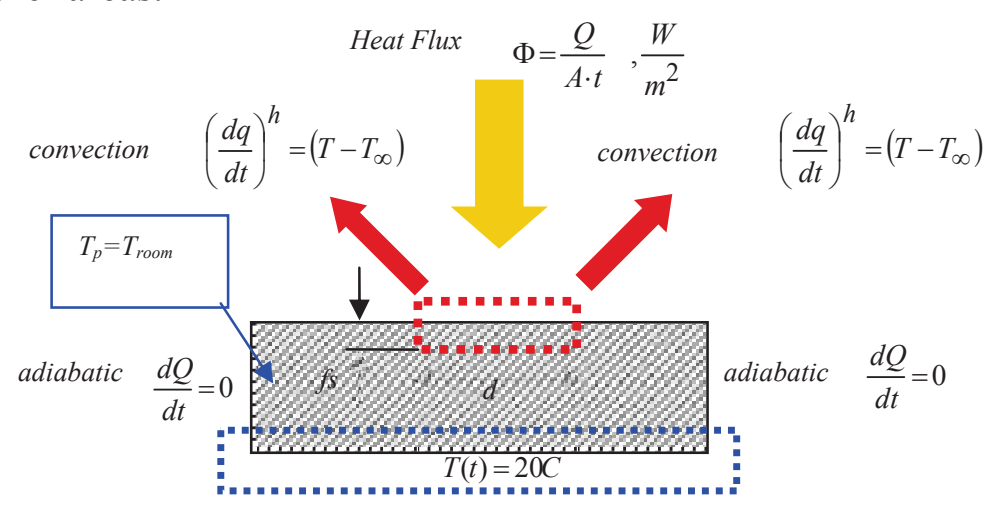

Fig .3. The physical model 
Consideration the additional load (not only due to the laser) resulted from the need to simulate the real conditions of the experiment. The value of convection of heat transfer coefficient for cooling the sample flowing air at room temperature, taken from the literature $\mathrm{h}=21 \mathrm{~W} /\left(\mathrm{m}^{2} \mathrm{~K}\right)[3]$.

The model samples with dimensions of $\mathrm{D}=0.2 \mathrm{~mm}$ and $\mathrm{h}=3.75 \mu \mathrm{m}$ consisted of 175000 knots and 161000 elements, which describe finite element mesh. All the elements were elements in the shape of Solid cubes with a height of $25 \mathrm{~nm}(2.5 \mathrm{E}-8 \mathrm{~m})$ to $250 \mathrm{~nm}$ and the edge of $0.250 \mu \mathrm{m}$ to $2.5 \mu \mathrm{m}$, with 8-point Gauss. Two planes of symmetry of the corresponding constraints are modelled. In place of concentrated load application grid.

\section{The results of numerical simulation}

Explicitly in the numerical analysis program, LS-Dyna performed with time step $\Delta \mathrm{t}=0.001 \mathrm{~ns}$ to $100 \mathrm{~ns}$ calculation time. As a result of the calculations, they will be provided with maps of stress, strain and temperature. The depth the ablation for the accepted power laser pulse was identified based on the criterion the temperature. Assumed that the ablation occurs in those areas of the model, which is as a result of vaporization material the temperature higher than the melting temperature of nickel superalloy $\mathrm{T}_{\text {melt }}$. The maximum depth the ablation, equal to $0.575 \mu \mathrm{m}$ was reached after a time $40 \mathrm{~ns}$ (Fig. 4). Temperature distribution in the zone the ablation is shown in Fig. 5.
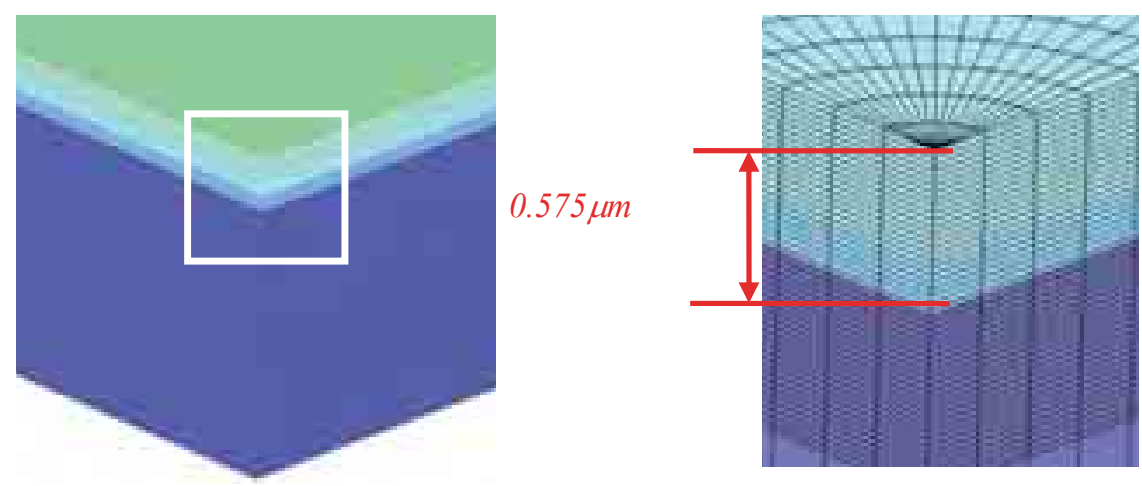

Fig. 4. The identification depth of the ablation (maps of temperature T> Tmelt): a - view of the model, $b-a$ magnified portion of the model with the selected depth the ablation

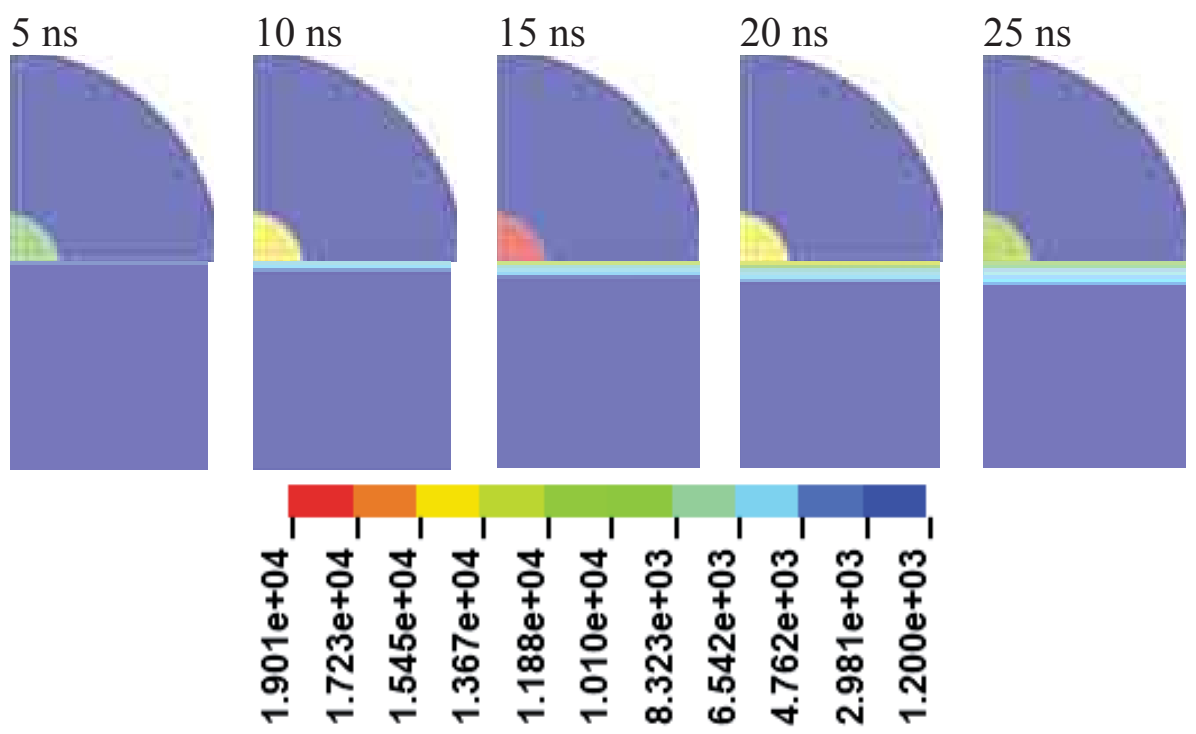

Fig. 5. The area where the temperature exceeded the melting temperature $T>T_{\text {melt }}\left({ }^{\circ} \mathrm{C}\right)$ in successive moments of loading (in the XY plane and the upper intersection $Z X$ ) 


\section{Conclusions}

The used material models (elastic-plastic with strengthening) well describe the material properties. Obtained results of calculations, such as stress and deformation maps, enable to observe the condition of the workpiece at any stage of the process. The results of FEM numerical simulations have confirmed their compatibility with the results of actual experiment carried out in the laboratory WAT, IE, ablative laser cleaning. The simulation calculations show that the depth of the ablation with a single laser impulse is $0.575 \mathrm{~m}$. The highest temperature was obtained in about $15 \mathrm{~ns}$ from the beginning of the laser impulse the sample load. Making changes to an already developed MES model, they give the freedom to shape desired properties of the treated material and improve existing treatment technologies.

\section{References}

[1] LS-Dyna user's manual, Vol. I, Livermore Software Technology Corporation, 2007.

[2] Kosiuczenko, K., Niezgoda, T., Napadłek, W., The identification of parameters the laser impulse LSP strengthening of aluminum alloy by the inverse method, Mechanik, 5-6, Warszawa 2008.

[3] Hsieh, H., Lin, J., Laser inducted vibration during pulsed laser forming, Science Direct Optics \& Laser Technology, 36, pp. 431-439, 2004. 